\title{
Incidencia de las enfermedades no transmisibles en los adultos mayores de la región Callao
}

\begin{abstract}
RESUMEN
El presente estudio tiene como objetivo examinar la incidencia de las enfermedades no transmisibles o no contagiosas e identificar a las principales dolencias que afectan la salud y la calidad de vida de la población adulta mayor residente en la región Callao, con el fin de conocer los cambios que se han producido en el patrón de causas de morbilidad, pasando de las enfermedades infecciosas o transmisibles hacia otras enfermedades no infecciosas o no transmisibles. En tal sentido, se diseñó un instrumento de recolección de datos, validado y confiable, que fue aplicado a 202 sujetos que forman parte de la muestra representativa de la población adulta mayor, de 60 y más años de edad, de sexo masculino y femenino respectivamente. La encuesta consta de 25 preguntas, es un cuestionario estructurado, genérico, directo, y los resultados obtenidos serán divulgados. El estudio concluye que la transición demográfica y la transición epidemiológica han producido cambios en la fecundidad, la mortalidad y en el patrón de enfermedades, predominando las no transmisibles.
\end{abstract}

Palabras clave: Enfermedades no transmisibles; enfermedades transmisibles; adultos mayores; transición epidemiológica; transición demográfica; morbilidad; calidad de vida.

\section{Incidence of non comunicable diseases in the elderly people from Callao region}

\begin{abstract}
The aim objective of this research is to examine the incidence of non-communicable or non-contagious diseases and to identify the main illnesses ailments that affect the health and quality of life for the elderly population who live in the Callao region, in order to know the changes that have occurred in the pattern of causes of morbidity, passing from infectious or transmissible diseases to non-infectious or non-communicable others. Due to this, a validated and reliable data collection instrument was designed, which was applied to 202 subjects that are part of the representative sample of people older than 60 years or older, between men and women. The survey, which consists of 25 questions, is a structured, generic and direct questionnaire, and the results obtained will be disclosed. This research concludes that the demographic and epidemiological transitions have made changes in fertility, mortality and the pattern of illnesses, predominating non-communicable ones
\end{abstract}

KeYwords: Non-communicable diseases; transmissible diseases; adult greater; demographic transition; epidemiological transition; morbidity; quality of life. 


\section{Introducción}

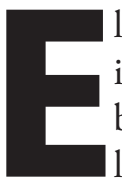
1 estudio de investigación trata el tema de la incidencia de las enfermedades no transmisibles en la población adulta mayor, residente en la región Callao. Esta situación es resultado de los procesos de la transición epidemiológica y demográfica, que en los últimos años, viene experimentando la población peruana. Es en este contexto que se han producido diferentes cambios demográficos relacionados con la mortalidad, la natalidad y con el proceso de envejecimiento de la población. De tal manera, que se ha observado cambios en los patrones de enfermedad, invalidez y muerte, pasando de la primacía de enfermedades transmisibles a una situación de predominio de las enfermedades no transmisibles o crónicas degenerativas, afectando seriamente a la salud de la población adulta mayor. Esta situación constituye un reto para el sistema de salud que tiene que atender a más personas afectadas por enfermedades como hipertensión, diabetes, enfermedades respiratorias crónicas, entre otras.

Según la Organización Mundial de la Salud (OMS), en este siglo se producirá el envejecimiento generalizado de la población con enfermedades no transmisibles, no infecciosas o crónicas degenerativas, como consecuencia de los procesos de transición demográfica y epidemiológica, que se producen con diferentes ritmos en todos los países del mundo. En Perú, en las últimas décadas se ha presentado un lento proceso de envejecimiento demográfico, como consecuencia de la disminución de la natalidad y del incremento en la supervivencia de las personas de 60 ańos de edad y más. Es así que se han producido cambios demográficos relacionados con la estructura por edad de la población, con las transiciones demográfica y epidemiológica y con las variaciones en la morbilidad, mortalidad y en la esperanza de vida de la población del Callao. En el proceso de la transición epidemiológica se observan cambios en el patrón de las enfermedades, predominando las no transmisibles, ocasionando incapacidad y la muerte de la población (Minsa, 2010).

\section{Objetivo general}

Examinar la incidencia de las enfermedades no transmisibles en la población adulta mayor de la región Callao.

\section{Importancia de la investigación}

El estudio se justifica porque permitirá conocer el proceso de transición epidemiológica, así como el estado de salud de los adultos mayores, respecto a las enfermedades crónicas-degenerativas. Este trabajo tiene el propósito de concitar la atención de las autoridades del sector salud de la región Callao para que propongan políticas públicas de salud orientadas hacia la prevención y control de las enfermedades no transmisibles en los adultos mayores para reducir la tasa de morbilidad y mortalidad temprana.

\section{Marco teórico}

\section{Aspecto geográfico de la región Callao}

El Callao fue creado el 20 de agosto de 1836. El 22 de abril de 1857 se le otorga el título de Provincia Constitucional del Callao, con rango de departamento. En su territorio se encuentra la capital regional y la sede del gobierno regional del Callao, además de las instalaciones del principal puerto marítimo del país, el Callao y en razón de su ubicación geoestratégica se encuentra en proceso de ampliación y modernización y va en camino a ser el HUB que inter conectará América Latina con el continente de Asia, y también están las instalaciones del Aeropuerto Internacional Jorge Chávez, es decir se convierte en la principal puerta de entrada al Perú y de salida. La ciudad del Callao, también es sede de las diferentes direcciones regionales de los ministerios que conforman la administración pública. En los últimos años, el Callao se ha caracterizado por su gran actividad industrial, comercial y residencial (Gobierno Regional del Callao, 2016).

\section{Localización}

El Callao se localiza en la región natural de la costa, en la parte central y occidental del Perú, entre las coordenadas geográficas: $11^{\circ} 47^{\prime} 50$ " y $12^{\circ} 07^{\prime} 30$ ” de Latitud Sur y los $77^{\circ} 04^{\prime} 40$ » y $77^{\circ} 11^{\prime} 40$ » de longitud Oeste.

\section{Limites}

Por el norte, con los distritos de Santa Rosa y Ancón; por el sur, con el distrito de San Miguel; por el este, con los distritos de Puente Piedra, San Martín de 
Porres y el Cercado de Lima y por el oeste, con el Océano Pacífico (Gobierno Regional del Callao. 2016).

\section{Extensión superficial, división política y altitud}

La superficie territorial es de $146.98 \mathrm{~km}^{2}$, incluyendo la superficie insular oceánica que abarca un área de $17.63 \mathrm{~km}^{2}$.

Según la división política-administrativa, la región Callao se divide en seis distritos. En el año 2014 se creó el distrito Mi Perú (Cuadro 1).

Cuadro 1. Región Callao: Distritos y superficie territorial en $\mathrm{km}^{2}$

\begin{tabular}{|l|c|c|}
\hline \multicolumn{1}{|c|}{ Distritos } & $\begin{array}{c}\text { Superficie } \\
\text { territorial km }{ }^{2}\end{array}$ & $\%$ \\
\hline Total & 146.98 & 100.00 \\
\hline Callao & 45.65 & 35.3 \\
Bellavista & 4.56 & 3.5 \\
Carmen de la Legua-Reynoso & 2.12 & 1.7 \\
La Perla & 2.75 & 2.1 \\
La Punta & 0.75 & 0.6 \\
Ventanilla & 73.52 & 56.8 \\
\hline
\end{tabular}

Incluye $17.63 \mathrm{~km}^{2}$ de superficie insular oceánica.

Fuente: INEI. Censos Nacionales de Población y Vivienda 2007.

La altitud varía, el territorio se eleva desde los 0 msnm, por el oeste, hasta los $540 \mathrm{msnm}$, por el este (Gobierno Regional del Callao, 2016).

\section{Aspecto físico}

\section{Relieve}

La región Callao se extiende en la región natural de la costa o chala. Aproximadamente el $60 \%$ de su superficie es plana y está por debajo de los 40 m.s.n.m., el resto del territorio presenta colinas áridas. Su relieve es plano y está emplazado en el valle aluvial de los ríos Rímac y Chillón, actualmente la mayor parte del área está urbanizada. En el valle aluvial hay presencia de acumulaciones detríticas y están limitados y en contacto con acumulaciones fluviales constituidos por cantos rodados transportados de zonas interandinas (Peñaherrera, 1969).

\section{Hidrografía}

El Callao ocupa la parte baja de las cuencas de los ríos Rímac y Chillón. Estos ríos que recorren el territorio de la región Callao se originan en la cordillera occi- dental de los Andes. Su dirección es de este a oeste, de corto recorrido y su caudal varía con las estaciones del ańo. Ambos ríos pertenecen a la vertiente del Pacífico. El río Chillón después de recorrer el distrito de Ventanilla desemboca en el océano Pacífico y el río Rímac lo hace después de recorrer el distrito del Callao. Ambos ríos conforman amplios valles aluviales, con suelos aptos para el desarrollo agrícola. El rio Rímac tiene gran importancia económica y es un factor importante para el desarrollo del Callao (Peñaherrera, 1986).

\section{Clima}

El tipo de clima que presenta el Callao es subtropical árido. Una particularidad del clima de la costa es su temperatura más baja que la correspondiente a su latitud. El Callao tiene una temperatura media anual de 19.2 grados (Pulgar Vidal, 1981). Sus características varían con las estaciones del año, con temperaturas máximas de $26^{\circ} \mathrm{C}$, la temperatura mínima es de $18.7^{\circ} \mathrm{C}$ y la temperatura promedio anual es de $18.2^{\circ} \mathrm{C}$, con excesiva humedad atmosférica, con precipitaciones tipo garúa, con un promedio de $7 \mathrm{~mm}$ al año, con vientos alisios suaves, con dirección Sur y Sur Este y con la presencia de nubes estratos que está muy vinculada con el proceso de inversión térmica (Gob. Reg. del Callao, 2016).

\section{Aspecto poblacional de la región Callao}

Los Censos del año 2007, revelan que la población censada de la región Callao fue de 876877 habitantes, de los cuales 430582 son varones, con un porcentaje de $49.1 \%$ y 446295 son mujeres, que representa el $50.9 \%$ de la población total. El distrito que presenta mayor población es el Callao, con 415888 habitantes (47.4\%) y el distrito con menor población es La Punta, con 4370 habitantes $(0.5 \%)$. La población económicamente activa es de 366706 , la proporción de la población urbana es de $100 \%$, con una densidad poblacional de 6061.3 $\mathrm{h} / \mathrm{km}^{2}$, y su esperanza de vida es de 77.7 (INEI, 2008).

La población adulta mayor, asciende a 80909 personas, de los cuales 39106 son varones, lo que representa el 48\% y 41803 son mujeres, con el 52.0\% de la población y con un índice de masculinidad de 93.5 (Cuadro 2). 
CUADRo 2. Región Callao: Población adulta mayor de hombres y mujeres por grupos quinquenales e índice de masculinidad

\begin{tabular}{|c|c|r|r|r|r|r|r|}
\hline $\begin{array}{c}\text { Grupos } \\
\text { quinquenales } \\
\text { de edad }\end{array}$ & Total & $\%$ & Hombres & $\%$ & Mujeres & $\%$ & IM \\
\hline Total & $\mathbf{8 0 9 0 9}$ & $\mathbf{1 0 0 . 0}$ & $\mathbf{3 9 1 0 6}$ & $\mathbf{4 8 . 0}$ & $\mathbf{4 1 8 0 3}$ & $\mathbf{5 2 . 0}$ & \\
\hline $60-64$ & 24877 & 30.7 & 12100 & 14.9 & 12777 & 15.8 & 94.7 \\
$65-69$ & 19051 & 23.5 & 9481 & 11.7 & 9570 & 1.8 & 99.1 \\
$70-74$ & 14705 & 18.1 & 7220 & 8.9 & 7485 & 9.3 & 96.5 \\
$75-79$ & 10768 & 13.3 & 5234 & 6.5 & 5534 & 6.8 & 94.6 \\
$80-84$ & 6547 & 8.1 & 2946 & 3.6 & 3601 & 4.5 & 81.8 \\
85 y más & 4961 & 6.1 & 2125 & 2.6 & 2836 & 3.5 & 74.9 \\
\hline
\end{tabular}

Fuente: INEI. Censos Nacionales 2007: XI de Población y VI de Vivienda.

Elaboración propia

\section{El adulto mayor}

En el año de 1982, la Primera Asamblea Mundial sobre Envejecimiento acordó considerar como adulto mayor a las personas de 60 y más años de edad. Lo mismo hizo la Organización Mundial de la Salud (OMS, 1982), que estableció el uso del término adulto mayor para referirse a las personas de $60 \mathrm{y}$ más años. En 1998, el Estado peruano, a través del D.S. N. ${ }^{\circ}$ 011-987-Promudeh, define al grupo de población Adultos Mayores, a las personas mayores de sesenta años (Mindes).

\section{Calidad de vida}

El término calidad de vida hace referencia a la evaluación subjetiva de la salud que simultáneamente valora aspectos objetivos y subjetivos de la persona, como la salud física, el estado psicológico, nivel de independencia, relaciones sociales, condiciones de entorno y creencias espirituales. Por su parte la OMS, en Ginebra, en 1966, define a la calidad de vida como «la percepción del individuo sobre su

Gráfico 1. Índice de masculinidad de la región Callao por grupos quinquenales de edad posición en la vida dentro del contexto cultural y sistema de valores en el que vive y en relación con sus objetivos, expectativas, estándares e intereses». La calidad de vida es la percepción que tienen las personas de que sus necesidades están siendo satisfechas o se les niegan oportunidades de auto realización y alcanzar la felicidad con independencia de su estado de salud y de las condiciones sociales y económicas. Por consiguiente, la calidad de vida es una definición que considera el grado de satisfacción que posee la persona con relación a su estado físico, emocional, social, vida familiar y el sentido que le da a su vida (Botero y Pico, 2007).

\section{Transición demográfica}

La transición demográfica es un proceso que transcurre entre dos situaciones o regímenes extremos: uno, inicial, de bajo crecimiento demográfico, con altas tasas de mortalidad y fecundidad, y otro final, con niveles también bajos en las respectivas tasas. Entre ambas situaciones de equilibrio se pueden identificar dos momentos principales. El primero, la tasa de crecimiento de la población aumenta como consecuencia del descenso de la mortalidad, y el segundo, el crecimiento disminuye, debido al descenso posterior de la fecundidad (Chesnais, 1986).

América Latina se encuentra en la fase de la disminución de la fecundidad, que se ha producido en forma acelerada, después de haber experimentado cambios en la mortalidad antes de 1950 (Chakiel y Martínez, 1993).

De acuerdo a la tipología del Centro Latinoamericano y Caribeño de Demografía (Celade), nuestro país está en plena transición demográfica, presentando natalidad y mortalidad moderada. En el Perú hay varias transiciones 
demográficas en curso, que están ligadas a las inequidades existentes en el desarrollo económico y social de cada uno de los departamentos. Lima Metropolitana se halla en la etapa avanzada de la transición demográfica, al presentar una natalidad y mortalidad baja, esto se traduce en un crecimiento natural bajo de la población, entre tanto, departamentos como Huancavelica y Apurímac, están en la etapa incipiente de la transición demográfica, con altas tasas de natalidad y mortalidad. Considerando las cifras que muestran los indicadores demográficos como la natalidad y mortalidad que presentan la región Callao, con tasas brutas de natalidad de 18.37 por mil y de mortalidad de 4.1 por mil, es decir con niveles bajos, nos indica que se encuentra en la fase de transición demográfica avanzada. Esto significa que en la región el desarrollo social y económico es significativo (INEI, 2002).

\section{Transición epidemiológica}

La transición epidemiológica es un proceso a largo plazo de las variaciones en las condiciones de salud de una población que incluyen cambios en los patrones de enfermedad, invalidez y muerte. Constituye un proceso dinámico y continuo, en el cual los modelos de salud y enfermedad de una sociedad se van transformando en respuesta a modificaciones más amplias de carácter demográfico, socioeconómico, tecnológico, político, cultural y biológico (OMRAN, 1971).

La transición epidemiológica evoluciona en fases que coinciden con la demográfica. Así en la fase temprana de la transición epidemiológica, cuando predomina la población joven, esta enferma y fallece principalmente por enfermedades infecciosas, traumatismos, envenenamientos y violencia. En la fase tardía, las personas se encuentran bajo la influencia de los factores de riesgo para enfermedades crónicas como consecuencia de vivir un mayor número de años determinando que sean estas sus principales causas de morbilidad y mortalidad (Minsa, 2010).

\section{Enfermedades transmisibles}

Según la OMS, son aquellas que se transmiten de un ser humano a otro, o de animales al hombre, y que son transportadas por insectos $\mathrm{u}$ otros vectores, o que son transmitidas por el aire que respiramos, el agua que bebemos o el suelo que pisamos.
Así en el período comprendido entre 1990 y 2006 se han producido cambios en el patrón de las enfermedades, observándose que las principales causas de muerte en el Callao fueron: causas externas (3.0\%), enfermedades transmisibles (31.0\%), enfermedades no transmisibles $(66.0 \%)$ y para el año 2006 se registraron los siguientes valores: causas externas (5.1\%), enfermedades transmisibles (19.9\%), y enfermedades no transmisibles (79.0\%) (Minsa, 2010).

\section{Enfermedades no transmisibles}

Son enfermedades no contagiosas, pero que son perjudiciales para la vida de cualquier persona que ha sido diagnosticada. Son aquellas que se padecen durante muchos ańos, ocasionan la muerte a largo plazo y siendo necesario un control médico periódico y tratamiento para toda la vida. Son enfermedades crónicas, de larga duración y de progresión lenta. Entre ellas tenemos las enfermedades cardiacas, los infartos, el cáncer, las enfermedades respiratorias y la diabetes, consideradas como las principales causas de mortalidad en el mundo. Teniendo en cuenta el envejecimiento de la población y los factores de riesgos, se calcula que para el año 2020, el $70 \%$ de las defunciones serán causadas por enfermedades no transmisibles (OMS, 2008).

Las enfermedades cardiovasculares, cerebros vasculares, diabetes mellitus, asma bronquial causan incapacidad y muerte en la población. La carga de las enfermedades no transmisibles no sólo incide sobre la calidad de vida de las personas afectadas y de sus familiares, sino también en la estructura socioeconómica del país (Minsa, 2010).

\section{Morbilidad}

La morbilidad señala la proporción de personas que enferman en un espacio y un periodo determinado. También permite comprender la evolución de una enfermedad, así como las razones de su aparición y las posibles soluciones (OMS, 2008).

\section{Métodos y técnicas utilizadas}

El tipo de investigación es básica, descriptiva, explicativa y transversal. Es un estudio mixto: cualitativo y cuantitativo. El estudio comprende el área geográfica de la región Callao. 
Según el INEI (2007), los datos del Censo revelan que la población adulta mayor de la región Callao fue de 80909 personas, es decir menor a 100000 sujetos, por consiguiente se ha considerado que el tamaño de la muestra y su representatividad, se obtenga mediante la aplicación de una fórmula para poblaciones finitas, con un nivel de confianza de 0,05 y con un error de 3\%. La muestra está conformada por 202 sujetos.

Para el estudio se empleó un instrumento, validado y confiable, que fue diseñado especialmente para medir la incidencia de las enfermedades no transmisibles en los adultos mayores, que fue aplicado a los 202 sujetos que forman parte de la muestra representativa de la población adulta mayor, de 60 y más años de edad, de sexo masculino y femenino y que residen en la región Callao. El trabajo de campo se realizó en el mes de junio del año 2012. Para la recolección de la información se contó con personal capacitado. A los encuestados se les seleccionó por el método aleatorio simple. Los criterios de inclusión considerados son los siguientes: que sean personas adultas mayores, que residen en la región Callao y acepten participar de manera voluntaria en el estudio. La encuesta consta de varias preguntas, es un cuestionario estructurado, genérico, directo, anónimo y los resultados obtenidos serán divulgados.

Después de la recolección de la información se procedió con el procesamiento de datos, para lo cual se creó una base de datos computarizada, usando el paquete Estadístico para las Ciencias Sociales SPSS, versión 17.

\section{Exposición, sistematización e interpretación de los resultados logrados}

La encuesta se aplicó a 202 personas adultas mayores que residen en la región Callao y que padecen de enfermedades no transmisibles y cuyas edades abarcan de 60 hasta los 96 años. Se observa que hay 92 encuestados (45.54\%) tienen entre 60 y 68 ańos, seguido de otros $71(35.15 \%)$ con 69 a 77 años, 29 $(14.36 \%)$ con 78 a 86 años y finalmente 10 (4.95\%) tienen 87 a 96 años (Gráfico 2).

Los datos muestran que conforme aumenta la edad de hombres y mujeres, la población adulta mayor disminuye.

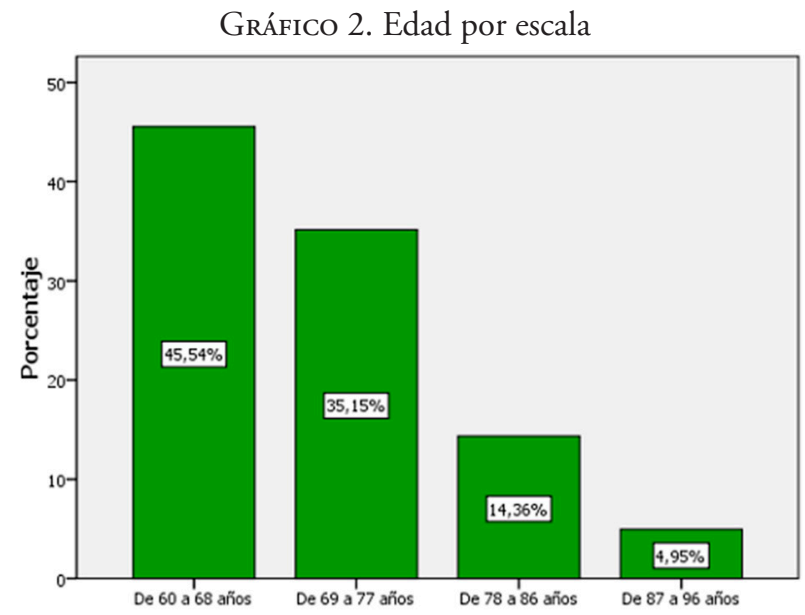

Fuente: INEI. Elaboración propia

Según los datos de los Censos 2007, la población adulta mayor, por grupos quinquenales de edad, por sexo e índice de masculinidad de la región Callao, revelan que la población total de adultos mayores es de 80909 (100\%), de los cuales 39100 (48.0\%) son hombres y 41803 (52.0\%) son mujeres, con un índice de masculinidad de 93.5 por cada 100 mujeres. Es decir que existe más mujeres (4.0\%) que hombres.

El grupo quinquenal de edad de 60 a 64 años, con 24877 adultos mayores, que representa el $30.7 \%$, de los cuales 12100 (14.9\%) son hombres y 12777 (15.8\%) son mujeres. El grupo de 65 a 69 ańos, con 19051 (23.5\%), de los cuales 9481 (11.7\%) son hombres y $9570(11.8 \%)$ son mujeres. El grupo de 70 a 74, con 14705 (18.1\%) de los cuales 7220 (8.9\%) son hombres y $7485(9.3 \%)$ son mujeres. Le sigue el grupo de 75 a 79 años, con 10768 (13.3\%), de los cuales 5234 (6.5\%) son hombres y $5534(6.8 \%)$ son mujeres. El grupo de 80 a 84 ańos, con 6547 (8.1\%), con 2946 (3.6\%) hombres y 3601 (4.5\%) mujeres. Finalmente el grupo de 85 y más años presenta 4961 adultos mayores, con el porcentaje de $6.1 \%$ de la población, siendo el grupo con menor población adulta mayor, con 2125 (2.6\%) hombres y 2836 (3.5\%) mujeres. Es decir se observa que conforme se avanza en edad, la población adulta mayor disminuye, tanto en cifras absolutas como en cifras relativas. También apreciamos el predominio de las mujeres, en todos los grupos quinquenales de edad de la población adulta mayor, debido a que las mujeres viven más años que los hombres, es decir, la esperanza de vida es mayor que la de los hombres (Cuadro 2) 
Cuadro 3. Región Callao

\begin{tabular}{|c|c|c|c|c|c|c|c|c|c|c|c|c|}
\hline & \multicolumn{2}{|c|}{$\begin{array}{c}\text { Tiene seguro de } \\
\text { salud }\end{array}$} & \multicolumn{2}{|c|}{ Vive solo } & \multicolumn{2}{|c|}{$\begin{array}{c}\text { Padece de alguna } \\
\text { enfermedad } \\
\text { crónica }\end{array}$} & \multicolumn{2}{c|}{$\begin{array}{c}\text { Recibe atención } \\
\text { médica }\end{array}$} & $\begin{array}{c}\text { Toma medicamen- } \\
\text { tos para controlar } \\
\text { su enfermedad }\end{array}$ & $\begin{array}{c}\text { Los medicamentos } \\
\text { los toma de por } \\
\text { vida }\end{array}$ \\
\hline & Abs & $\%$ & Abs & $\%$ & Abs & $\&$ & Abs & $\%$ & Abs & $\%$ & Abs & $\%$ \\
\hline Total & 202 & 100 & 202 & 100 & 202 & 100 & 202 & 100 & 202 & 100 & 202 & 100 \\
\hline Sí & 140 & 63.3 & 34 & 16.8 & 196 & 97.0 & 181 & 89.6 & 182 & 91.0 & 124 & 61.4 \\
\hline No & 62 & 30.7 & 30.7 & 83.2 & 6 & 3.0 & 21 & 10.4 & 18 & 9.0 & 78 & 38.6 \\
\hline
\end{tabular}

Fuente: Encuesta. Elaboración propia.

Cuadro 4. Región Callao

\begin{tabular}{|c|c|c|c|c|c|c|c|c|c|c|}
\hline & $\begin{array}{c}\text { El dinero le alcanza } \\
\text { para pagar } \\
\text { sus consultas } \\
\text { médicas }\end{array}$ & \multicolumn{2}{|c|}{$\begin{array}{c}\text { Le alcanza el dinero } \\
\text { para comprar sus } \\
\text { medicamentos }\end{array}$} & \multicolumn{2}{|c|}{$\begin{array}{c}\text { La enfermedad } \\
\text { afecta su calidad de } \\
\text { vida }\end{array}$} & \multicolumn{2}{|c|}{$\begin{array}{c}\text { Su enfermedad } \\
\text { le ha producido } \\
\text { discapacidad física }\end{array}$} & \multicolumn{2}{c|}{$\begin{array}{c}\text { Recibe ayuda } \\
\text { económica de su } \\
\text { familia }\end{array}$} \\
\hline & Abs & $\%$ & Abs & $\%$ & Abs & $\%$ & Abs & $\%$ & Abs & $\%$ \\
\hline Total & 202 & 100 & 202 & 100 & 202 & 100 & 202 & 100 & 202 & 100 \\
\hline Sí & 91 & 45.0 & 93 & 67.3 & 136 & 67.3 & 25 & 25.7 & 122 & 60.4 \\
\hline No & 111 & 55.0 & 109 & 54.0 & 66 & 32.7 & 150 & 74.3 & 80 & 39.6 \\
\hline
\end{tabular}

Fuente: Elaboración propia.

Cuando se analiza el índice de masculinidad, IM de la región Callao, se observa un comportamiento diferenciado en los grupos quinquenales de edad. De esta manera, vemos que en el grupo quinquenal de edad de 60 a 64 años el IM es de 94.7 hombres por cada 100 mujeres. En el grupo de 65 a 69, la tendencia es a subir, presentando un IM de 99.1 por cada 100 mujeres y desciende en el grupo de 70 a 74 ańos, con 96.5, y continúa el descenso que apreciamos en los grupos de 75 a 79 años, con 94.6. En el grupo de 80 a 84 años, con 81.8 , y en el grupo de 85 y más años, con 74.9 por cada 100 mujeres. En este grupo se aprecia que el índice de masculinidad es más bajo con respecto a los otros grupos de edad. Este comportamiento se debe a que la esperanza de vida de los hombres se ha reducido considerablemente (Cuadro 2).

Otro de los resultados del estudio tiene que ver con el padecimiento de enfermedades crónicas. Se observa que, de los 202 encuestados, 196 (97.03\%) padece alguna enfermedad crónica, mientras que el $2.97 \%$ no lo padece. Estas cifras nos indican que la mayoría de la población sufre de enfermedades no transmisibles (Gráfico 3).

En referencia a cómo perciben su calidad de vida, se observa el siguiente resultado: 109 encuestados $(54.0 \%)$ perciben que su calidad de vida es regular, $65(32.2 \%)$ perciben que su calidad de vida es buena. Otros 17 (8.4\%) perciben que es mala, 9
Gráfico 3. ¿Padece de alguna enfermedad crónica?

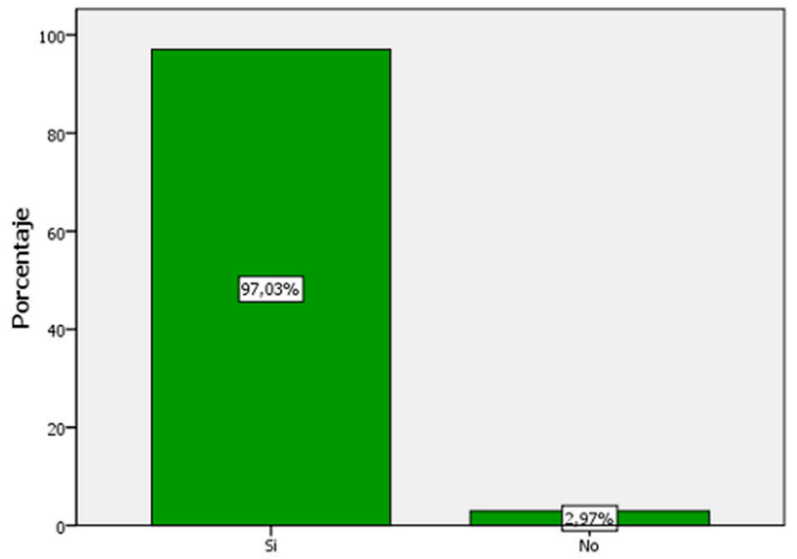

Fuente: INEI. Elaboración propia

(4.5\%) perciben que es muy buena y $2(1 \%)$ perciben que su calidad de vida es muy mala (Gráfico 4).

Con relación a la percepción de estado de salud, se aprecia que el $55.4 \%$ percibe que es regular, el $26.2 \%$ es buena, el $12.4 \%$ es mala, el $5.0 \%$ es muy buena y el $1.0 \%$ percibe que su estado de salud es muy mala (Gráfico 5).

En la región Callao, se produce el proceso de envejecimiento de la población, observándose en la pirámide poblacional, tanto en la base, que es reducida, como en la cima, de la pirámide donde se aprecia el aumento relativo de los adultos mayores como con- 
Región Callao. Percepción de la calidad de vida y estado de salud

Gráfico 4. Percepción de calidad de vida

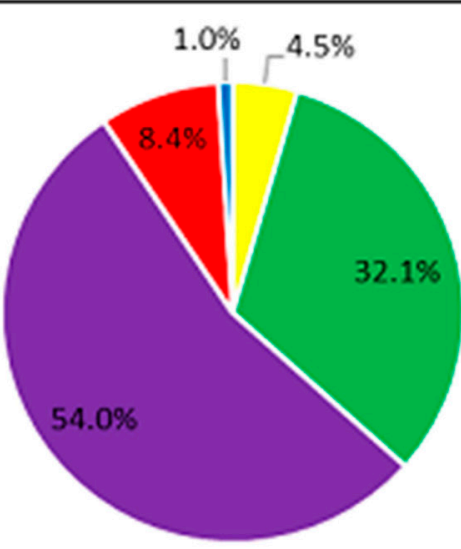

Muy Buena = Buena " Regular = Mala = Muy Mala

Fuente: INEI. Elaboración propia

secuencia del incremento de la esperanza de vida al nacer (Gráfico 6).

De los 202 adultos mayores encuestados, 140 encuestados $(69.3 \%)$ sí tiene seguro de salud y 62 (30.7\%) no lo tiene. En cuanto a si vive solo, 34 (16.8\%) contestaron que si viven solos, en cambio $182(83.2 \%)$ no viven solos. La gran mayoría, 196, con el porcentaje de $97.0 \%$ padece de alguna enfermedad crónica y solo 6 personas, con el $3.0 \%$ no lo padece. 181 encuestados con el 89.6\% sí recibe atención médica. Por el contrario $21(10.4 \%)$ no recibe atención médica. En cuanto a la toma de medicamentos para controlar la enfermedad, 182 (91.0\%) sí los toman y $18(9.0 \%)$ no los toman. También se observa que 124 , con el $61.4 \%$, toman los medicamentos de por vida y $78(38.6 \%)$ no lo toman de por vida (Cuadro 3).

Los encuestados acuden a establecimientos de salud del Minsa y EsSalud para controlar su enfermedad y realizar su consulta en medicina general.

De los 202 encuestados, 91, (45.0\%) manifestaron que el dinero si les alcanza para pagar sus consultas médica y $111(55.0 \%)$ no les alcanza. También $93(46.0 \%)$ contestaron que el dinero si les alcanza para comprar sus medicamentos y 109 (54.0\%) no les alcanza. La mayoría de los encuestados, 136 representados con el porcentaje 67.3\%, manifestaron que la enfermedad sí afecta su calidad de vida y 66 (32.7\%) no afecta su calidad de vida. En cuanto a si
Gráfico 5. Percepción de estado de salud

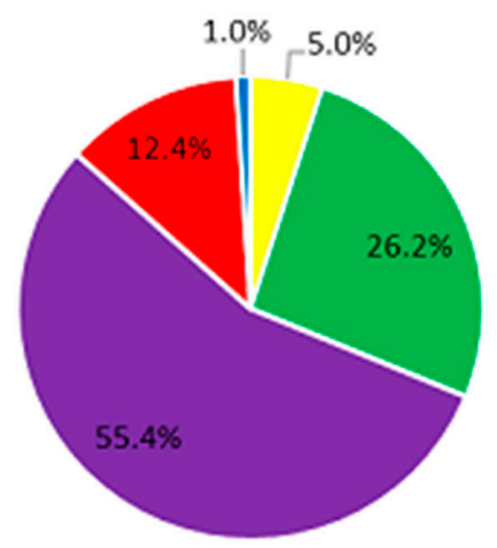

Muy Buena $=$ Buena $=$ Regular $=$ Mala $=$ Muy Mala

GrÁfico 6. Región Callao:

Pirámide de población, según censos 2007

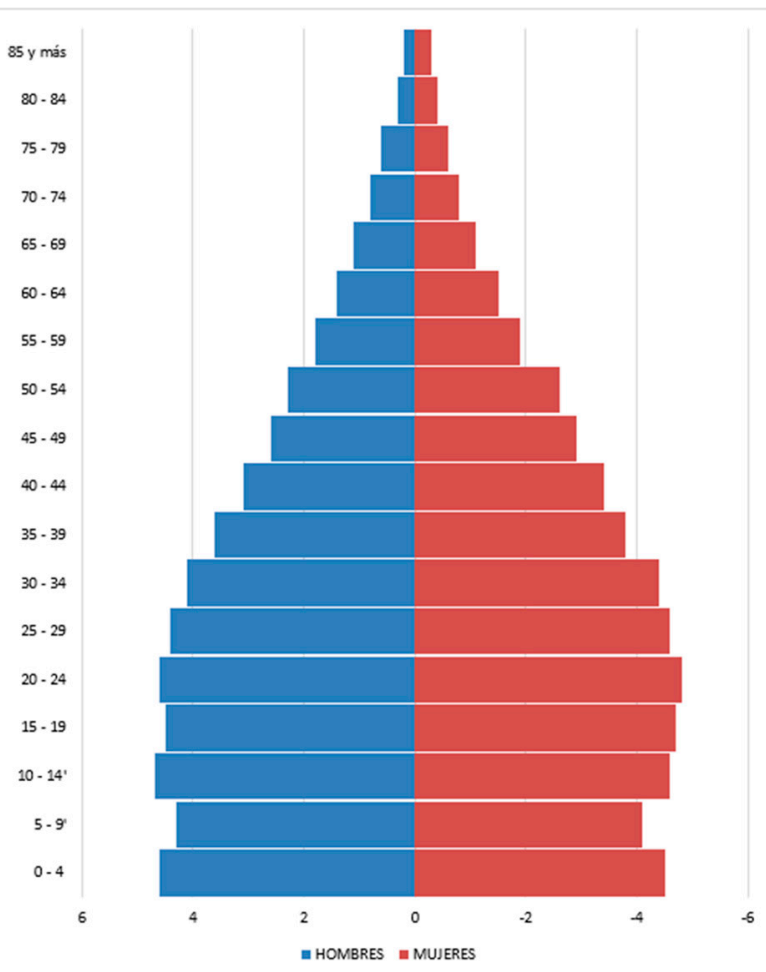

Fuente: INEI. Censos Nacionales de Población y Vivienda 2007. Elaboración propia

su enfermedad le ha producido discapacidad física, $52(25.7 \%)$ contestaron que sí y $150(74.3 \%)$ no. Con relación a si recibe ayuda económica de su fa- 
Gráfico 7. Su estado de salud le impide realizar otras actividades

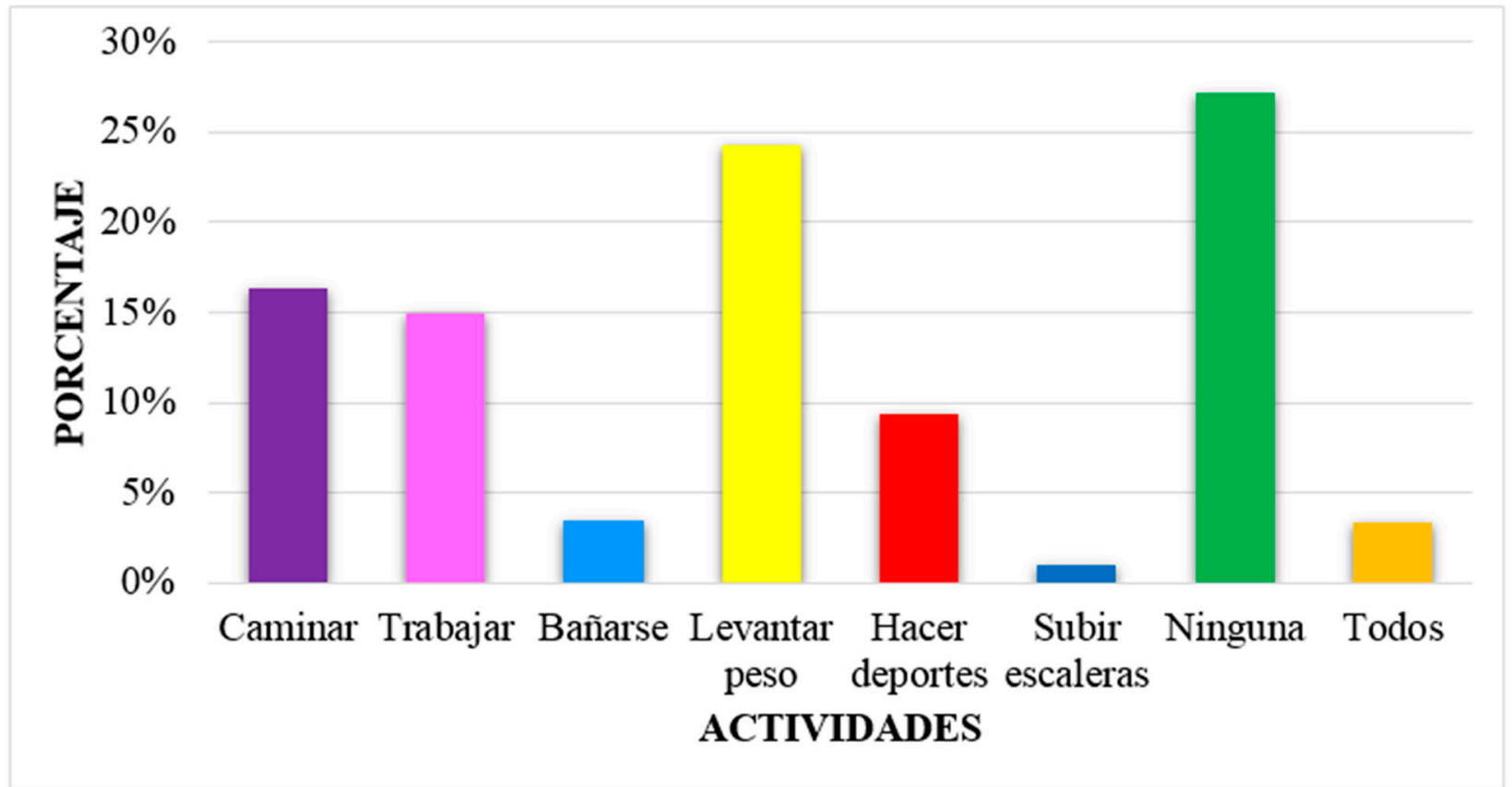

Fuente: Encuesta. Elaboración propia

Gráfico 8. Nivel de instrucción

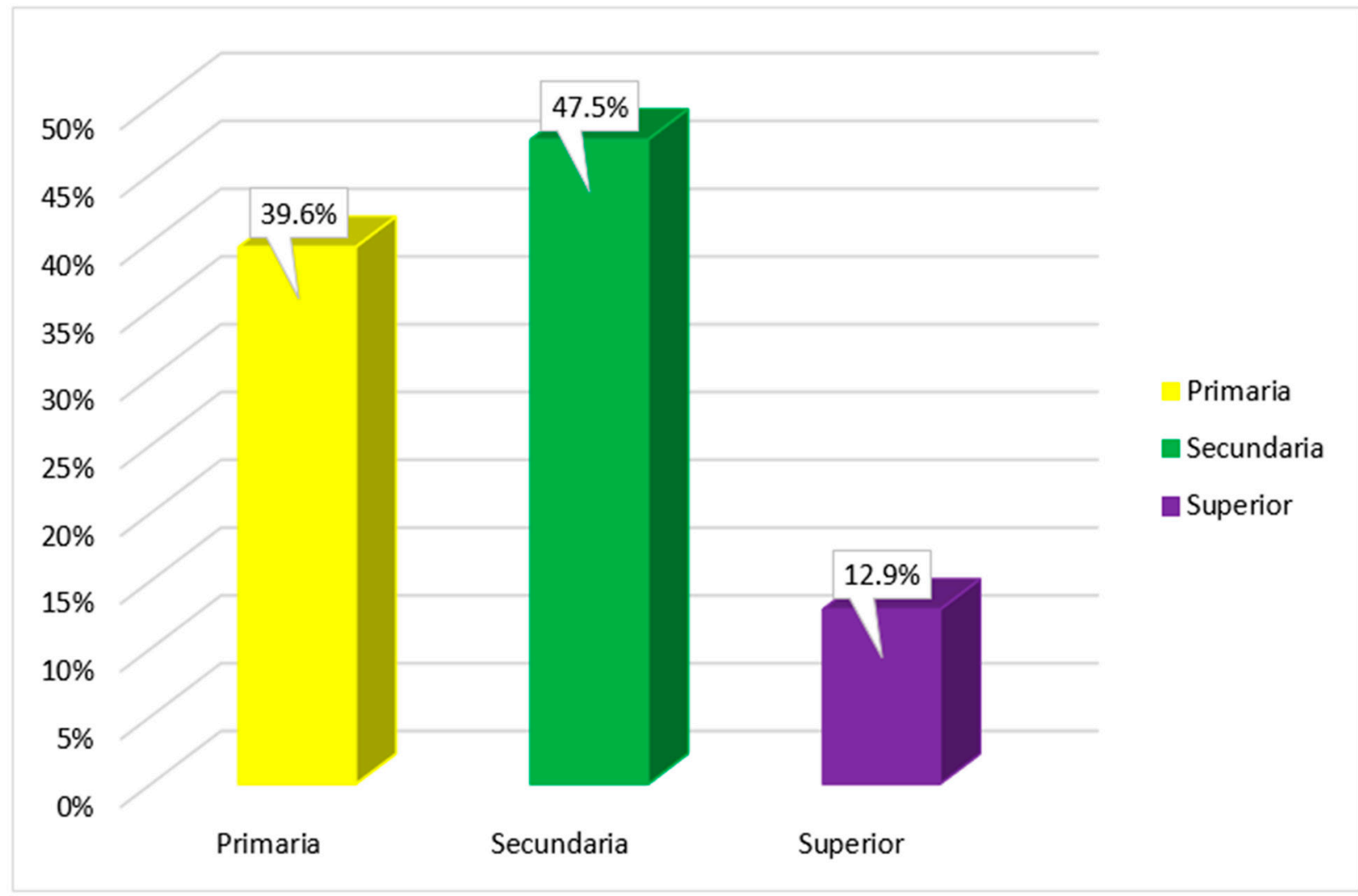

Fuente: Encuesta. Elaboración propia 
Gráfico 9. Estado civil

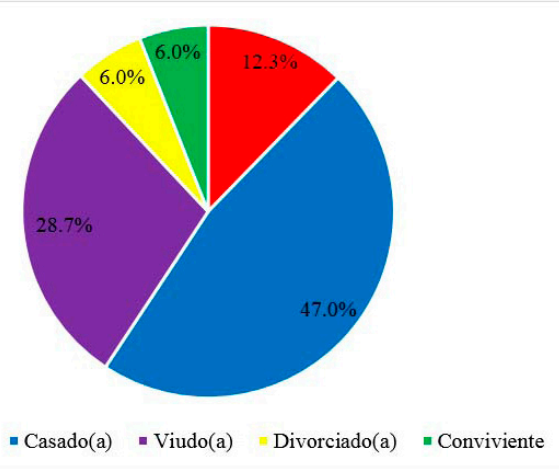

- Soltero(a) - Casado(a) =Viudo(a) = Divorciado(a) = Conviviente

Fuente: Encuesta. Elaboración propia

GrÁfico 10. Situación laboral

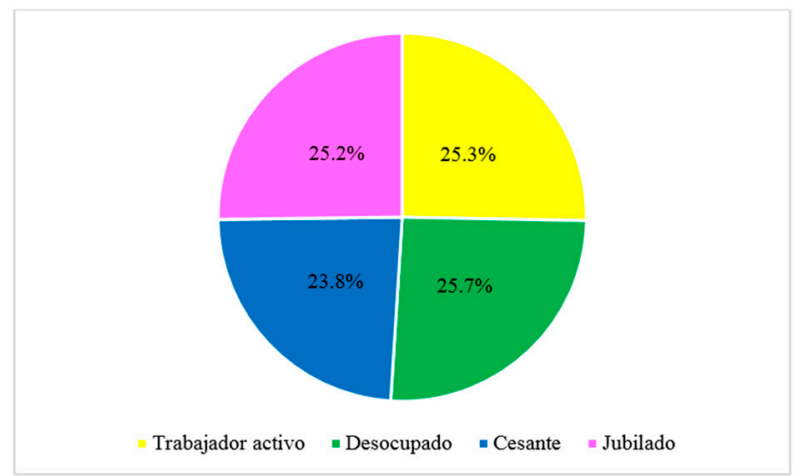

Fuente: Elaboración propia

milia, $122(60.4 \%)$ contestaron que sí y 80 (39.6\%) manifestaron que no. (Cuadro 4).

Con respecto a que su estado de salud le impide realizar otras actividades como caminar, 33 encuestados, (16.3\%) manifestaron que su estado de salud les impide caminar, 30 sujetos (14.9\%) revelaron que les impide trabajar, a $7(83.5 \%)$ les impide bañarse y a 49 (24.3\%) les impide levantar peso. Otros 19 (9.4) les impide hacer deportes. 2 sujetos $(1.0 \%)$ están impedidos de subir escaleras. A 55 personas, $(27.2 \%)$ su estado de salud no les impide realizar ninguna de las actividades indicadas y 7 encuestados (3.4\%) están impedidos de realizar todas las actividades señaladas. (Gráfico 7).

En cuanto al nivel de instrucción de los encuestados observamos que $80(39.6 \%)$ tienen nivel de instrucción primaria, $96(47.7 \%)$ poseen instrucción secundaria, y finalmente 25 encuestados, con el $12.4 \%$, con educación superior (Gráfico 8 ).

En cuanto al estado civil de los encuestados distinguimos que $25(12.4 \%)$ son solteros 95 son casados y representan el $47.0 \%$, seguido de 58 encues- tados que son viudos equivalente al $28.7 \%$. Otros 12 con el $6.0 \%$, son divorciados y 12 (6.0\%), son convivientes (Gráfico 9).

Con relación a la situación laboral de los encuestados vemos que 51 y con un porcentaje de $25.3 \%$ son trabajadores activos, 52 (25.7\%), están desocupados, $48(23.8 \%)$ son cesantes y 51 encuestados con el 25.2\% son jubilados (Gráfico 10).

\section{Conclusiones y recomendaciones}

\section{Conclusiones}

El proceso de transición demográfica ha producido modificaciones, disminuyendo las tasas de fecundidad y la mortalidad respectivamente, reflejándose en la estructura por edad de la población y en el aumento de la esperanza de vida al nacer. La región Callao se encuentra en la etapa de transición demográfica avanzada.

La transición epidemiológica en la región Callao es un proceso que ha producido variaciones en la salud de la población, observándose un cambio en el patrón de las enfermedades, predominando las enfermedades no transmisibles, afectando así la salud y la calidad de vida de los adultos mayores.

Por consiguiente, la causa de la morbilidad y la mortalidad de la población adulta mayor son por enfermedades no transmisibles, como la hipertensión arterial, la diabetes, las enfermedades respiratorias crónicas, enfermedades cardiovasculares, entre otras, que han incrementado la tasa de morbilidad y mortalidad por enfermedades crónicas.

Los datos de la encuesta muestran que el $97.03 \%$ de la población adulta mayor padece de alguna enfermedad no transmisible, y que toman medicamentos de por vida. También perciben que su estado de salud y calidad de vida es regular. Sólo el $2.97 \%$ no sufren de enfermedades crónicas y perciben su estado de salud y calidad de vida como buena o muy buena.

La enfermedad le ha producido discapacidad física al $25.7 \%$ de la población adulta mayor y el $74.3 \%$ no tiene discapacidad física.

Con relación a la población adulta mayor por sexo, se observa el predominio de las mujeres, con un $52.0 \%$ y de varones con $48.0 \%$ y un IM de 93.5 hombres por cada 100 mujeres. 
La tendencia del proceso de envejecimiento de la población de la región Callao va en aumento constantemente, haciéndose visible en la base y en la cima de la pirámide, es decir que la población infantil (base) disminuye progresivamente y la población de $60 \mathrm{y}$ más ańos de edad (cima) aumenta constantemente.

\section{Recomendaciones}

Mejorar e incrementar la infraestructura de salud de la región Callao para la atención médica de los adultos mayores.

Realizar tareas de prevención y control de enfermedades no transmisibles en la región Callao.

Difundir información concerniente a los centros de atención y salud para que la población adulta conozca los servicios geriátricos que ofrece la región Callao.

Ayudar económicamente y brindar atención médica a la población que no cuenta con los recursos económicos suficientes para su atención médica y para la compra de sus medicamentos.

Dar a conocer información nutricional e incentivar a la población afectada por enfermedades no transmisibles a que realice ejercicios físicos para mejorar su calidad de vida y su estado de salud.

\section{Bibliografía}

Botero de Mejía, Beatriz E., Pico Merchán, María E. (2007d). Calidad de vida relacionada con la salud (CVRS) en adultos mayores de 60 años. Una aproximación teórica. Hacia la promoción de la salud. Volumen 12. Colombia.
Chackiel, Juan y Martínez, Jorge. (1993). Transición demográfica en América Latina y el Caribe desde 1950. Volumen I, primera parte, IV Conferencia Latinoamericana de Población. México.

Chesnais, J. (1986). La transition démographique, étapes, formes, implications. París:PUF/INED.

Gobierno Regional del Callao. (2016). Atlas geográfico de la Provincia Constitucional del callao. Callao.

INEI, FNUAP (2002). Estudio de la población peruana 2002. Lima.

INEI (2008). Censos Nacionales 2007, XI de Población y VI de Vivienda. Perfil sociodemográfico del Perú. Lima.

Ministerio de la Mujer y Desarrollo Social. Plan nacional para las personas adultas mayores 2006-2010. Lima. Perú.

Ministerio de Salud (2010). Análisis de la situación de salud del Perú. Lima.

Omran, Abdel. (1971). The epidemiology transition; a theory of the epidemiology of population change.

Organización de Naciones Unidas. (1982). Asamblea mundial sobre envejecimiento. Viena.

Organización Mundial de la Salud. (1966). Foro mundial de la salud. Ginebra.

Organización Mundial de la Salud (1984). Aplicaciones de la epidemiología al estudio de ancianos. Ginebra.

Organización Mundial de la Salud (2008). Prevención y control de las enfermedades no transmisibles: aplicación de la estrategia mundial. Ginebra.

Organización Panamericana de la Salud (2003). Análisis de las condiciones de salud de las personas adultas mayores en América Latina y El Caribe.

Peñaherrera del Águila, Carlos. (1969). Geografía General del Perú. Lima.

Pulgar VIDAL, Javier. (1981). Las ocho regiones naturales del Perú. Lima. 